\title{
Subpopulations of natural killer-T-like cells before and after surgical treatment of laryngeal cancer
}

\author{
JANUSZ KLATKA ${ }^{1}$, EWELINA GRYWALSKA ${ }^{2}$, ANNA HYMOS ${ }^{1}$, EWELINA KRASOWSKA ${ }^{2}$, \\ MICHAE MIELNIK', DOROTA SIWICKA-GIEROBA ${ }^{2}$, JUSTYNA MARKOWICZ ${ }^{2}$, \\ PIOTR TROJANOWSKI ${ }^{1}$,WITOLD OLSZAŃSKI ${ }^{1}$, JACEK ROLIŃSKI ${ }^{2}$
}

${ }^{1}$ Department of Otolaryngology and Laryngeal Oncology, Medical University of Lublin, Lublin, Poland

${ }^{2}$ Department of Clinical Immunology and Immunotherapy, Medical University of Lublin, Lublin, Poland

\begin{abstract}
Introduction: Tumours connected with head and neck comprise about $5 \%$ of all tumours. The most frequent histological type of laryngeal carcinoma is squamous cell carcinoma. Different research projects suggest that the role of T lymphocytes might be significant in tumour development. iNKT cells are a new subpopulation of $T$ cells and show cytotoxic activity against tumours. iNKT cells participate in modulating the function of other cells which have anti-tumour properties and secrete cytokines, which have pro-inflammatory and anti-inflammatory effects. In animal models the significance of iNKT cells in various diseases including cancer was shown.

Aim of the study: The aim of this study was to determine the percentages of iNKT cells, CD161+ cells, CD161-cells, iNKT CD4+ cells, and iNKT CD8+cells, NK cells, NKT-like cells, and T cells subsets present in peripheral blood of patients with laryngeal cancer before and two months after the tumour resection, in comparison to healthy volunteers.

Materials and methods: This study included material from laryngeal patients who were treated at the Department of Otolaryngology and Laryngological Oncology (Medical University of Lublin) between 2012 and 2013. A total of 50 patients (40 men and 10 women) aged between 45 and 77 years (median age: 60 years) were enrolled. Based on the TNM classification, the patients were classified as having stage I-IV laryngeal cancer. The control group was composed of 15 healthy volunteers (12 men and three women) aged between 43 and 82 years (median age: 61 years). The protocol of the study was approved by the Local Bioethical Committee at the Medical University of Lublin.

Peripheral blood samples $(15 \mathrm{ml})$ from the basilic vein were collected by venipuncture using sterile, sodium heparin-treated tubes (20 units per $\mathrm{ml}$ of blood) and used for cytometric analyses.

Results: iNKT cells were analysed among $T C D 3+$ cells. The percentage of $C D 3+$ and $C D 3+C D 4+$ $T$ cells before tumour resection was higher than in the control group, but the increase of CD3+T cells was not significant. The $T C D 3+C D 4+/ T C D 3+C D 8+$ cell ratio was significantly higher than in healthy donors. After tumour resection a decreased percentage of $C D 3+C D 4+T$ cells but an increased percentage of $C D 8+C D 3+T$ cells was noted. The TCD3+CD4+ / TCD3+CD8+ cell ratio was significantly higher in patients before and after the surgery than in the control group. The amount of NKT-like cells increased after resection and was significantly higher than in the control group.

Conclusions: Our study exhibited the change in percentage of iNKT, NK, NKT-like cells, and T lymphocytes after tumour resection in patients with laryngeal cancer. The research explains the contribution of those cells in immunological response against tumour.
\end{abstract}

Key words: laryngeal cancer, T cells, iNKT cells, head and neck tumors.

(Centr Eur J Immunol 2017; 42 (3): 252-258)

\section{Introduction}

Tumours connected with head and neck comprise about $5 \%$ of all tumours. The most frequent histological type of laryngeal carcinoma is squamous cell carcinoma
$[1,2]$. Different research projects suggest that a role of $\mathrm{T}$ lymphocytes might be significant in tumour development [3]. iNKT cells are a new subpopulation of T cells and show cytotoxic activity against tumours [4]. iNKT cells

Correspondence: Janusz Klatka M.D., PhD, Professor, Department of Otolaryngology and Laryngeal Oncology, Medical University of Lublin, Jaczewskiego 8, 20-090 Lublin, Poland tel./fax. +48 817244518 / +48 8172445 17, e-mail: janusz.klatka@wp.pl Submitted: 7.07.2016; Accepted: 18.09.2016 
comprise $0.005 \%-0.2 \%$ of CD3+ T cells [5]. Generally, the definition of an NKT cell is a CD3 $+\mathrm{T}$ cell that expresses $\mathrm{T}$ cell receptor and NK cell molecules on its surface (such as CD161) [5, 6]. NKT cells were divided into type I, type II, and NKT-like cells [6]. In contrast to conventional T cells, NKT cells are able to recognise a cell's lipid antigens. Type I is called "invariant" NKT cells (iNKT cells) because of the expression of a semi-invariant TCR $\alpha$ chain with a V $\alpha 24$ J $\alpha 18$ gene segment. This type has the ability to recognise $\alpha$-galactosylceramide ( $\alpha$-GalCer, KRN7000 - a prototypic glycolipid antigen) and ganglioside antigens (GD3), which are, respectively, exogenous and endogenous glycolipid antigens $[4,7,8]$. The second NKT cell type are non-invariant NKT cells, which do not express the V $\alpha 14$ J $\alpha 18$ TCR $\alpha$-chain and do not have the possibility to recognize $\alpha$-GalCer. NKT-like cells are a T-cell subpopulation with memory phenotypes. Zrazilova Dubska et al. showed disease-free survival in patients with breast tumours that were associated with high level of NKT-like cells $[4,7]$.

iNKT cells participate in modulating the function of other cells that have anti-tumour properties. A commitment of iNKT cells to immune control is connected with indirect and direct involvement [9]. NKT cells connect innate and adaptive immunity [10]. iNKT cells secrete cytokines, which have pro-inflammatory and anti-inflammatory effects $[4,11]$. In animal models the significance of iNKT cells in various diseases including cancer has been shown. iNKT may be associated with the pathogenic role in infectious diseases, autoimmunity, and cancer $[8,10]$. Many tumour cells decrease levels of cancerous antigens, major histocompatibility complex, and costimulatory molecules. Cancer cells show the ability to produce transforming growth factor- $\beta$ (TGF- $\beta$ ), interleukin (IL)-10, or prostaglandin E-2 (PGE-2), which are suppressive molecules [12]. Those cells respond to the presence of cancer cells. However, iNKT cells are impaired and their functionality is abnormal $[4,10,13]$. In patients with various malignant diseases a decreased number and reduced function of iNKT cells was observed upon activation [9].

The available methods of treatment do not lead to the expected results [3, 14]. Recognition of components of the immune system the influence of which leads to development of tumours allows introduction of the most effective immunotherapy [15]. Surgery is a classical procedure, but this method does not provide satisfying effects [9]. Surgery and radiotherapy, however, empower positive results of treatment in early stages of the disease [1].

The aim of this study was to determine the percentages of iNKT cells, CD161+ cells, CD161- cells, iNKT CD4+ cells, and iNKT CD8+ cells, NK cells, NKT-like cells, and $\mathrm{T}$ cells subsets present in peripheral blood of patients with laryngeal cancer before and two months after the tumour resection in comparison to healthy volunteers.

\section{Material and methods}

This study included material from laryngeal patients who were treated at the Department of Otolaryngology and Laryngological Oncology (Medical University of Lublin) between 2012 and 2013. A total of 50 patients (40 men and 10 women) aged between 45 and 77 years (median age: 60 years) were enrolled. Based on the TNM classification, the patients were classified as having stage I $(n=4)$, stage II $(n=13)$, stage III $(n=22)$, or stage IV laryngeal cancer $(n=11)$. The control group was composed of 15 healthy volunteers (12 men and three women) aged between 43 and 82 years (median age: 61 years).

None of the enrolled individuals had undergone blood transfusions, suffered from infection, or had been taking antibiotics or other drugs with known influence on the immune system for a month before the examination. Patients with a history of allergic diseases were excluded from the study. The protocol of the study was approved by the Local Bioethical Committee, at the Medical University of Lublin.

Peripheral blood samples $(15 \mathrm{ml})$ from the basilic vein were collected by venipuncture using sterile, sodium heparin-treated tubes (20 units per $\mathrm{ml}$ of blood) and used for cytometric analyses.

Fresh peripheral blood was incubated with a set of monoclonal antibodies: anti-45 FITC/anti-14 PE, anti-iNKT FITC, anti- CD161 PE-Cy5, anti-CD3 PE-Cy5, anti-CD4 PE, anti-CD8 PE, anti-CD3 FITC/CD16+ CD56PE, anti-CD3 FITC/anti- CD19 PE, and anti-CD4 FITC/CD4 PE/ CD3 PerCP (BD Pharmingen, United States). The samples were deprived of erythrocytes by addition of a lysing solution (FACS Lysing Solution, Becton Dickinson, United States). The immunophenotype of peripheral blood cells was determined with a FACSCalibur flow cytometer (Becton Dickinson, United States) equipped with an argon laser emitting at $488 \mathrm{~nm}$. The results were analysed with CellQuestPro software (Becton Dickinson, United States).

\section{Statistical analysis}

Statistical analysis was conducted with Statistica 7.1 PL software (StatSoft, United States). The fractions of identified cells were expressed as mean $\pm \mathrm{SD}$, and median and range. Mann-Whitney U-test were used for intergroup comparisons, and Wilcoxon test was used for comparison of differences between patients and healthy donors. The differences were considered significant at $p<0.05$.

\section{Results}

iNKT cells were analysed among T CD3+ cells. The frequencies of iNKT cells were significantly lower in laryngeal cancer patients before tumour resection $(0.13 \pm 0.10 \%$; median $0.09 \%$; min. $0 \%$; max. $0.44 \%$; $p=0.000395 ;$ Fig. 1A) and after tumour resection $(0.19$ 

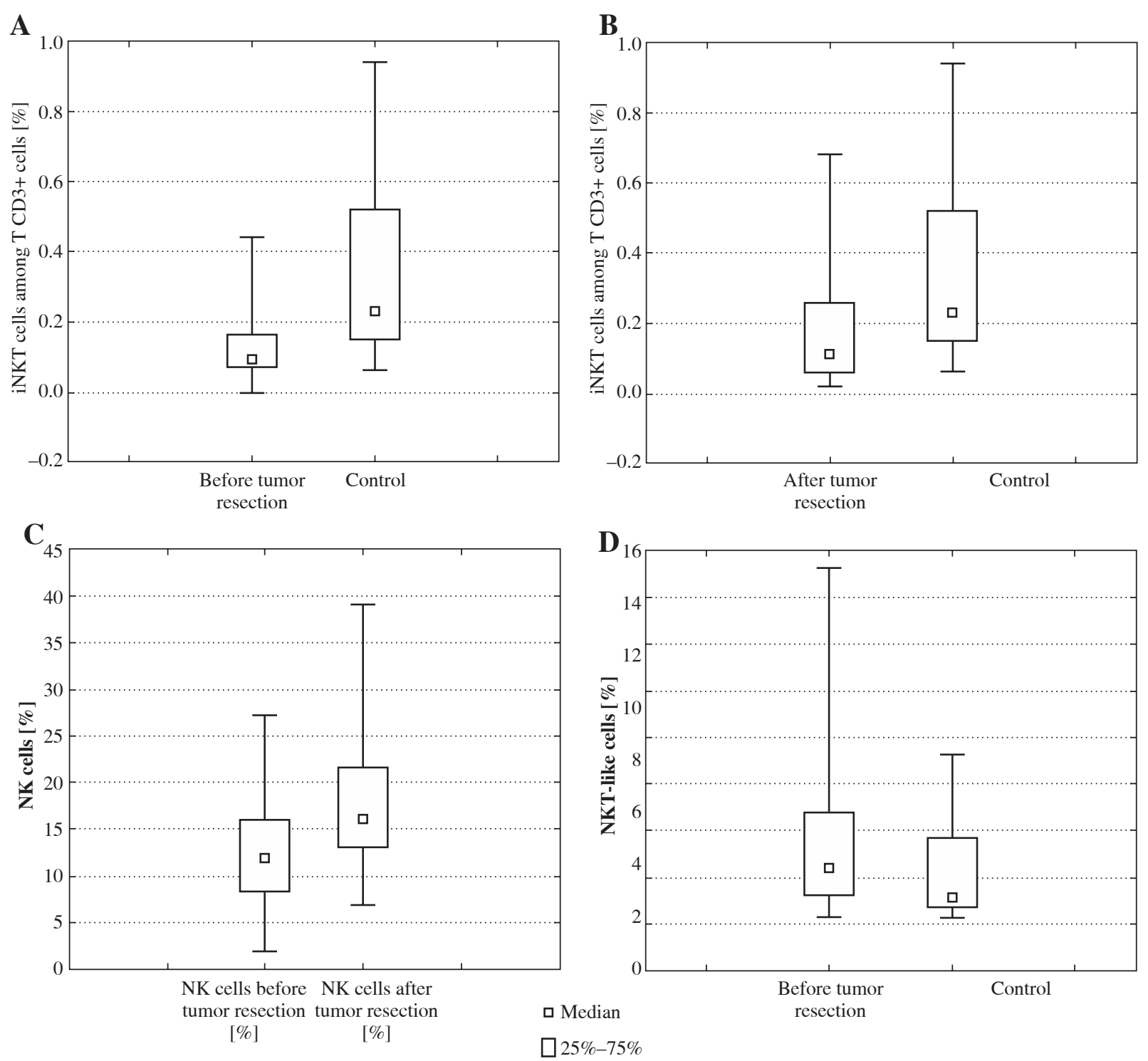

I Min.-Max.

Fig. 1. A) Percentage of iNKT cells among T CD3+ before tumor resection in comparison with control $(p=0.000395)$. B) Percentage of iNKT cells among T CD3+ after tumor resection in comparison with control $(p=0.01824)$. C) Percentage of NK cells among T CD3+ cells before tumor resection in comparison with percentage of NK cells after tumor resection $(p=0.00941)$. D) Percentage of NKT-like cells before tumor resection in comparison with control $(p=0.0346)$

$\pm 0.18 \%$; median $0.11 \%$; $\min .0 .02 \%$; $\max .0 .68 \%$; $p=$ 0.01824 ; Fig. 1B) in comparison with the control group (0.37 $\pm 0.12 \%$; median $0.23 \%$; min. $0.06 \%$; max. $0.94 \%)$. We did not observe statistically significant differences in the percentages of CD161+, CD4+, and CD8+ cells among iNKT cells between the study and control groups.

The mean percentage of NK cells was significantly higher in laryngeal cancer patients after cancer tissue resection (18.17 $\pm 7.74 \%$; median $15.99 \%$; $\min .6 .78 \%$; $\max$. $39.02 \%)$ than before $(12.61 \pm 5.79 \%$; median $11.72 \%$; min. 1.76\%; max. 27.07\%; $p=0.00941$; Fig. 1C).
We observed higher frequencies of NKT-like cells in patients before tumour resection $(3.38 \pm 2.88 \%$; median $2.42 \%$; min. 0.27\%; $\max .15 .21 \%$; $p=0.035$; Fig. 1D) in comparison with percentage of NKT-like cells in healthy donors $(1.99 \pm 1.93 \%$; median $1.11 \%$; min. $0.29 \%$; max. $7.27 \%)$. The frequencies of NKT-like cells were higher in study patients after tumour resection $(3.94 \pm 3.80 \%$; median $2.65 \%$; $\min .0 .43 \%$; $\max .17 .96 \% ; p=0.037$; Fig. $2 \mathrm{~A}$ ) than before this procedure $(3.38 \pm 2.88 \%$; median $2.42 \%$; $\min .0 .27 \%$; $\max .15 .21 \%)$. 

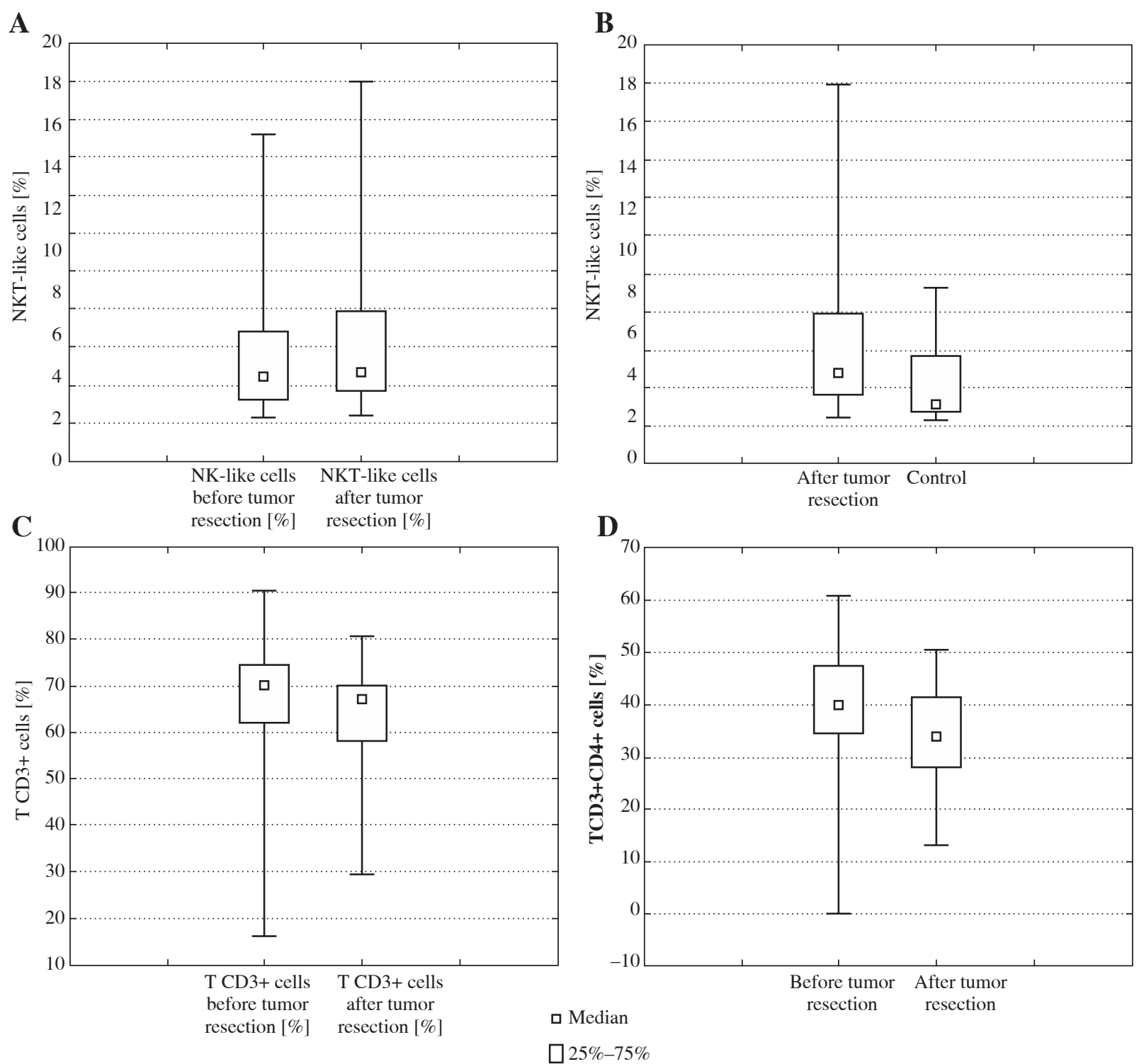

I Min.-Max.

Fig. 2. A) Percentage of NKT-like cells before tumor resection in comparison with percentage of NKT-like cells after tumor resection $(p=0.03704)$. B) Percentage of NKT-like cells after tumor resection in comparison with control ( $p=$ 0.0414). C) Percentage of T CD3+ cells before tumor resection in comparison with percentage of T CD3+ cells after tumor resection $(p=0.03955)$. D) Percentage of T CD4+ cells among CD3+ cells before tumor resection in comparison with percentage of those cells after tumor resection $(p=0.0038)$

Our study revealed also that the percentages of NKTlike cells were higher in patients after tumour resection than in the control group ( $p=0.04$; Fig. 2B).

The frequencies of T CD3+ cells in patients before tumour resection (67.63 $\pm 11.70 \%$; median $69.81 \%$; min. $15.99 \%$; max. $90.47 \%$ ) were significantly higher than after the resection $(62.99 \pm 12.04 \%$; median 67.0\%; min. 29.39\%; max. 80.57\%; $p=0.03955$; Fig. 2C). We found statistically significantly higher percentages of $\mathrm{T} \mathrm{CD} 3+/ \mathrm{CD} 4+$ cells in patients before tumour resection $(39.79 \pm 11.05 \%$; median $39.88 \%$; min. $19 \%$; max. $60.81 \%)$ than after $(34.21 \pm 9.45 \%$; median $33.78 \%$; min. 13.03\%; max. 50.4\%; $p=0.0038$; Fig. 2D).

Our research revealed also that in laryngeal cancer patients after tumour resection, the percentages of $\mathrm{T} \mathrm{CD} 3+/$ $\mathrm{CD} 8+$ cells were higher than before the procedure $(33.06$ $\pm 10.88 \%$; median $33.44 \%$; $\min .7 .61 \%$; $\max .49 .14 \%$ vs. $29.70 \pm 8.98 \%$; median $29.62 \%$; $\min$. $11.79 \%$; $\max$. $49.14 \% ; p=0.01855$; Fig. 3A). 

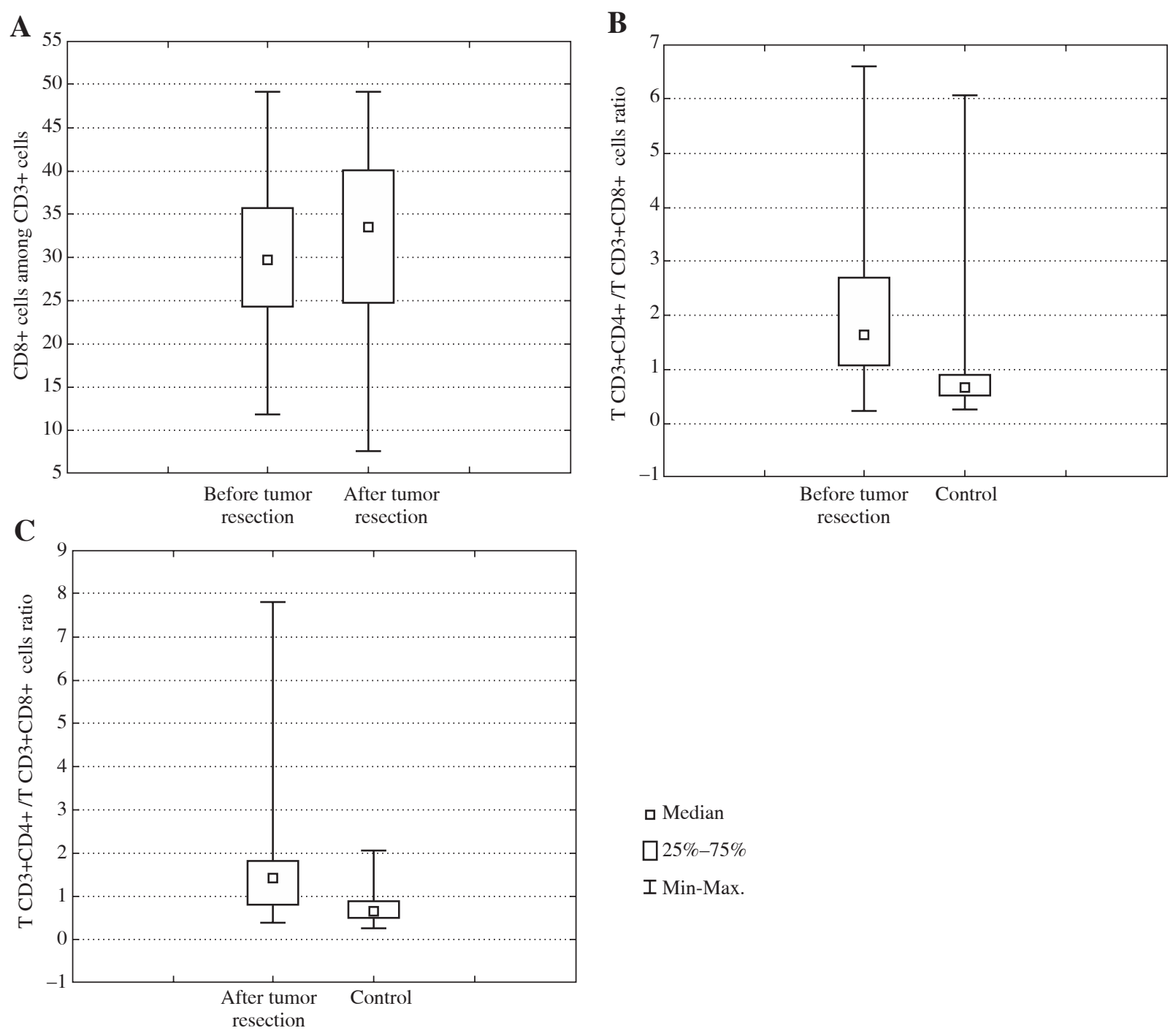

口 Median

$\square 25 \%-75 \%$

I Min-Max.

Fig. 3. A) Percentage of T CD8+ cells among CD3+ cells before tumor resection in comparison with percentage of those cells after tumor resection $(p=0.01855)$. B) T CD3+CD4+/T CD3+CD8+ cells ratio before tumor resection in comparison with control $(p=0.000426)$. C) T CD3+CD4+ / T CD3+CD8+ cells ratio after tumor resection in comparison with control $(p=0.00212)$

Moreover, the T CD3+CD4+/T CD3+CD8+ cell ratio was higher before tumour resection in the study group (1.91 \pm 1.33 ; median 1.62; min. 0.24; max. 6.59) and after this procedure $(1.97 \pm 1.84$; median 1.44 ; min. 0.4 ; max. $7.83)$ in comparison to the control group $(0.8 \pm 0.47$; median 0.66 ; min. 0.28 ; max. 2.05; $p=0.000426$; Fig. 3B and $p=0.00212$; Fig. 3C, respectively).

\section{Discussion}

The iNKT subpopulation was divided into two subsets. This division is connected with expression of CD4 and CD8 surface antigens [7]. We can divide this subpopula- tion into CD4+ and CD4-CD8- cells (double negative) and a small population of CD8+ cells. iNKT cell division on subpopulations of cells is connected with different functions. CD4+ iNKT secretes cytokines such as IL-4, GMCSF, IFN- $\gamma$, IL-13, and TNF-a [6], so final CD4+ NKT cells produce Th1 and Th2 cytokines. CD4-NKT cells, CD4-CD8-, and CD8+ NKT cells produce Th1 cytokines $[16,17]$. The predominant majority of CD4+ iNKT cells occur in foetal and neonatal blood; however, CD4-iNKT cells are found in peripheral blood [6]. CD4+ NKT cells activate NK cells, B cells, and CD4+ T cells whereas CD8+ NKT cells activate CD8+ T cells and NK cells [18]. 
Only type II NKT cells have suppressing properties, but other subtypes show opposite capabilities. Two phases have been exhibited in NKT activation. The early phase is connected with production of Th1 and Th2 cytokines, whereas the second phase is responsible for secretion of proinflammatory cytokines - IFN- $\gamma$ and TNF by NKT cells [18].

Among cells connected with protection against tumour we can distinguish CD8+ and CD4+ T lymphocytes [5]. Other cells that have an important function in immune surveillance against cancers are NK cells. The role of NK cells is associated with their ability to kill tumour cells [19].

NKT cells are able to protect against tumour growth. In various types of cancers, a reduction in the amount of NKT cells and in their functional activity was observed [20].

Cytotoxic properties of iNKT cells are connected with the occurrence of perforin, Fas/FasL, and TRAIL- molecules, which induce death of cells. On the other hand, iNKT can activate other cells: NK cells, CD8+ T cells, and $\mathrm{DC}$, and then those cells participate in killing the tumour. After activation, iNKT cells produce IFN- $\gamma$, which subsequently activates $\mathrm{NK}$ and CD8+ T cells. CD8+ T cells are responsible for recognising the tumour antigens, and NK cells exhibit an anti-tumour response. Probably, the presence of iNKT cells can inhibit the suppressive functions of certain cells such as macrophages or myeloid-derived suppressor cells [21]. Regulatory functions have been attributed to NKT cells because of their secretion of IL-4, which leads to the Th2 response [22].

Because of the diverse functions of NKT cells, there is a possibility that two subsets of NKT cells can be distinguished. Type II NKT cells have the suppressing properties, but another subset of NKT cells can show anti-tumour properties. NKT cells can present cytotoxicity similar to that of CD8+ T cells. This ability divides NKT cells into cytotoxic and non-cytotoxic subtypes [17].

Laryngeal cancer is connected with the occurrence of extraoesophageal reflux. In the mucosa of patients with extraoesophageal reflux increased levels of NKT cells, CD8+ $\mathrm{T}$ cells, and CD1d molecules can be found [23]. Molling et al. reported a reduced number of T cells and NK cells in patients with laryngeal cancer in comparison to healthy controls with a high statistical significance.

Molling et al. showed a notable decrease of iNKT cells $/ 10^{6} \mathrm{~T}$ cells. However, the results were not statistically significant. Tumours are said to be able to produce immunosuppressive cytokines [24]. The engagement of iNKT cells in the anti-tumour response in patients with cancer can explain the reduction of their number, despite which, iNKT cells retain the ability to produce IFN- $\gamma$ [21].

On the other hand, the decrease of iNKT cell numbers can be explained by apoptosis of those cells, and their migration and accumulation on the tumour site. However, tumour leads to the occurrence of a defective NKT cell population, which is caused by the reduction of CD1d ex- pression of myeloid DCs, participate in correct activation of NKT cells [24].

In our research the numbers of CD161+, CD4+, and CD8+ cells in the iNKT population were evaluated. We did not find a significant difference between percentage of those cells before surgery and after. CD161 is expressed on T, NK, and iNKT cells [16]. CD161 is a receptor expressed on NK cells and $25 \%$ of blood T lymphocytes and on NK cells. CD161 is associated with memory phenotype of T lymphocytes. The majority of NKT cells express CD161+. On the other hand, the minority of CD161- NK cells or subset $\mathrm{T}$ cells express CD56 antigen [25]. We observed a lower percentage of CD161+, CD4+, and CD8+ among iNKT cells in healthy individuals in comparison to examined patients. The percentage of CD161- cells was higher in the control group, but this assessment lacked statistical significance. Tye increase in the number of iNKT cells after resection was not significant, but the percentage of iNKT cells in comparison with healthy controls was significantly decreased.

Anti-tumour response is connected with antigen-specific CD4+ or CD8+T cells [21].

Kuss et al. showed a decreased absolute number of $\mathrm{CD} 3+, \mathrm{CD} 4+$, and CD8+ T cells in patients with laryngeal cancer in comparison with the control group, but Kuss et al. did not obtain similar results in their comparison of the percentage of $\mathrm{T}$ cell subsets. The investigators did not show a change in the percentage of CD3+ and CD4+, CD8+ subpopulations of T cells [27]. Chen et al. reported that total levels of lymphocyte numbers were significantly decreased in comparison to the control group. The decrease of CD4+ T cells was not substantial nor statistically important. The level of CD8+ T lymphocytes was higher in patients with cancer than in healthy individuals [11]. Li et al. obtained the same result as Kuss and Chen, but the slight increase of CD8+ T cells showed no statistical importance [14].

Our research indicates that the percentage of CD3+ and $\mathrm{CD} 3+\mathrm{CD} 4+\mathrm{T}$ cells before tumour resection was higher than in the control group, but the increase of CD3+ T cells was not significant. The percentage of $\mathrm{T} \mathrm{CD} 3+/ \mathrm{CD} 8+$ cells before tumour resection was slightly lower than in the control group. The T CD3+CD4+/T CD3+CD8+ cell ratio was significantly higher than in healthy donors. After tumour resection a decreased percentage of CD3+CD4+ $\mathrm{T}$ cells but an increased percentage of CD8+ T cells was noted. Each of the changes in those subpopulations were statistically significant, but the most important difference was connected with the decrease of CD3+/ CD4+ T cells. The comparison of $\mathrm{T} \mathrm{CD} 3+\mathrm{CD} 4+/ \mathrm{T} \mathrm{CD} 3+\mathrm{CD} 8+$ cell ratios between patients suffering from cancer and healthy individuals is interesting because of its considerable statistical dependence and the fact that it is significantly higher before and after the surgery than in the control group. 
NKT-like cells are connected with immunological activation caused by a tumour, and they start a cytotoxic immune response against it [16]. The protective role of NKT-like cells against cancer has been described in breast cancer and chronic lymphocytic leukaemia. Zdrazilova-Dubska showed higher numbers of NKT-like cells in patients with cancer than in the reference population [7].

NKT-like cells were another subpopulation of NKT cells that was investigated. Their numbers increased after resection and were significantly higher than in the control group.

Kuss et al. showed a reduction of NK cell numbers [27], and Kou et al. reported decreased activity of those cells in comparison with healthy subjects [12].

In our research the percentage of NK cells before tumour resection was lower than in the control group but increased after the procedure.

In summary, our study exhibited a change in percentage of iNKT, NK, NKT-like cells, and T lymphocytes after tumour resection in patients with laryngeal cancer. The research explains the contribution of those cells in immunological response against tumour.

This work was supported by research grant no. NN403 104240 from the Polish State Funds for Scientific Research and no. 2011/01/N/NZ6/01762 from the National Science Centre.

The authors declare no conflict of interest.

\section{References}

1. Reuter CW, Morgan MA, Eckardt A (2007): Targeting EGF-receptor-signalling in squamous cell carcinomas of the head and neck. Br J Cancer 96: 408-516.

2. Pantel M, Guntinas-Lichius O (2012): Laryngeal carcinoma: epidemiology, risk factors and survival. HNO 60: 32-40.

3. Kardasz-Ziomek M, Scierski W, Namyslowski G, et al. (2012): Long term results of partial laryngectomies in patients suffering from laryngeal cancer. Otolaryngol Pol 66: 46-50.

4. Klatka J, Grywalska E, Wasiak M, et al. (2016): The percentage of iNKT cells among other immune cells at various clinical stages of laryngeal cancer. Postepy Hig Med Dosw 70: 392-399.

5. Sharma AA, Chew L, Ladd M, et al. (2011): Ex vivo purification and characterization of human invariant natural killer T cells. J Immunol Methods 373: 1-7.

6. Rogers PR, Matsumoto A, Naidenko O, et al. (2004): Expansion of human Valpha24+ NKT cells by repeated stimulation with KRN7000. J Immunol Methods 285: 197-214.

7. Zdrazilova- Dubska L., Valik D, Budinska E, et al. (2012): NKT- like cells are expanded in solid tumour patients. Klin Oncol 25: 2S21-2S25.

8. Terabe M, Berzofsky JA (2014): The immunoregulatory role of type I and type II NKT cells in cancer and other diseases. Cancer Immunol Immunother 63: 199-213.

9. Metelitsa LS (2004): Flow cytometry for natural killer T cells: multi-parameter methods for multifunctional cells. Clin Immunol 110: 267-276.
10. Grywalska E, Markowicz J, Siwicka D, et al. (2013): Natural killer and natural killer T-like cells in splenectomised patients. Centr Eur J Immunol 38: 372-379.

11. Chen W, Wang X, Tao R (2011): Analysis of serum T-lymphocyte subsets and NK cell activity in patients with squamous cell carcinoma of pharynx and larynx [Abstract]. Lin Chung Er Bi Yan Hou Tou Jing Wai Ke Za Zhi 25: 1105-1107.

12. Kou F, Qi S (2001): Changes of NK cell's activity and T lymphocyte subpopulation in peripheral blood of patients with laryngocarcinoma [Abstract]. Lin Chuang Er Bi Yan Hou Ke Za Zhi 15: 505-506.

13. Sakakura K, Chikamatsu K, Takahashi K, et al. (2006): Maturation of circulating dendritic cells and imbalance of $\mathrm{T}$ cell subsets in patients with squamous cell carcinoma of the head and neck. Cancer Immunol Immunother 55: 151-159.

14. Li Y, Che Z, Liang M (1997): Study on the immune state of patients with laryngeal carcinoma [Abstract]. Lin Chuang Er Bi Yan Hou Ke Za Zhi 11: 69-72.

15. Ritoe SC, de Vegt F, Scheike IM, et al. (2007): Effect of routine follow-up after treatment for laryngeal cancer on life expectancy and mortality: results of a Markov model analysis. Cancer 109: 239-247.

16. Klatka J, Grywalska E, Klatka M, et al. (2013): Expression of CD200 and CD200R regulatory molecules on the CD83+ monocyte-derived dendritic cells generated from patients with laryngeal cancer. Folia Histochem Cytobiol 51: 59-65.

17. Perlabo E, Alonso C, Solana R (2007): Invariant NKT and NKT-like lymphocytes: Two different $\mathrm{T}$ cell subsets that are differentially affected by ageing. Exp Gerontol 42: 703-708.

18. Balato A, Unutmaz D, Gaspari AA (2009): Natural killer T cells: an unconventional T-cell subset with diverse effector and regulatory functions. J Invest Dermatol 129: 1628-1642.

19. Ambrosino E, Berzofsky JA, Terabe M (2008): Regulation of tumor immunity: the role of NKT cells. Expert Opin Biol Ther 8: 725-734.

20. Moretta L, Montaldo E, Vacca P, et al. (2014): Human natural killer cells: origin, receptors, function, and clinical applications. Int Arch Allergy Immunol 164: 253-264.

21. Metelitsa LS (2011): Anti-tumor potential of type-I NKT cells against CD1d-positive and CD1d-negative tumors in humans. Clin Immunol 140: 119-129.

22. Motohashi S, Okamoto Y, Yoshino I, et al. (2011): Anti-tumor immune responses induced by iNKT cell-based immunotherapy for lung cancer and head and neck cancer. Clin Immunol 140: 167-176.

23. Carnaud C, Lee D, Donnars O, et al. (1999): Cutting edge: Cross-talk between cells of the innate immune system: NKT cells rapidly activate NK cells. J Immunol 163: 4647-50.

24. Molling JW, Langius JA, Langendijk JA, et al. (2007): Low levels of circulating invariant natural killer $\mathrm{T}$ cells predict poor clinical outcome in patients with head and neck squamous cell carcinoma. J Clin Oncol 25: 862-868.

25. Singh AK, Gaur P, Das SN (2014): Natural killer T cell anergy, costimulatory molecules and immunotherapeutic interventions. Hum Immunol 75: 250-260.

26. Takahashi T, Dejbakhsh-Jones S, Strober S (2006): Expression of CD161 (NKR-P1A) defines subsets of human CD4 and CD8 T cells with different functional activities. J Immunol 176: 211-216.

27. Kuss I, Hathaway B, Ferris RL, et al. (2004): Decreased absolute counts of T lymphocyte subsets and their relation to disease in squamous cell carcinoma of the head and neck. Clin Cancer Res 10: 3755-3762. 